\title{
Diagnostic and counselling difficulties using a fully comprehensive screening protocol for families at risk for tuberous sclerosis
}

\author{
L I AL-GAZALI*, R J ARTHUR $\dagger$, J T LAMB $†$, H M HAMMER , \\ 'T P COKER , P N HIRSCHMANN\&, J GIBBS $\uparrow$ AND R F MUELLER* \\ From the Departments of Genetic Counselling*, Radiologyt, and Ophthalmology $\neq$, The General Infirmary at \\ Leeds; §The Dental Hospital at Leeds; and Ithe Department of Cardiology, Killingbeck Hospital, Leeds.
}

SUMmaRY Tuberous sclerosis (TS) results from an autosomal dominant gene which exhibits variable expression and reduced penetrance. Although there are well established diagnostic criteria for TS, examination of first degree relatives can cause diagnostic problems with consequent difficulties in genetic counselling.

Using an extensive, non-invasive protocol consisting of skin examination with Wood's lamp, cranial CT scan, specialist ophthalmological and dental examination, skeletal survey, and echocardiography, we have examined 56 first degree relatives of persons with TS. These consisted of 40 parents and seven sibs from 25 sporadically affected families and nine persons from seven multigeneration families.

In seven of the apparently sporadically affected families, three mothers had echocardio- 8 graphical findings consistent with one or more rhabdomyoma. In another, the mother's renalo ultrasound showed evidence of single cysts in both kidneys. In a fifth family, the father had suggestive but not diagnostic features of TS on the cranial CT scan and skeletal survey. In the $\rightleftharpoons$ sixth family, the mother was found to have atypical calcification on CT scan. In a seventh instance a sib from a two generation family had echocardiographical evidence of a rhabdomyoma.

Even though the proband in three of the sporadically affected families presented with fits, developmental delay, and depigmented patches, and therefore did not strictly fulfil the diagnostic criteria for TS, two mothers were found to have evidence of rhabdomyomata on echocardiography and the brother of the third had typical depigmented patches.

Although the presently accepted diagnostic criteria for TS may not allow one to make a definitive diagnosis of TS in these relatives, we recommend that an extensive screening protocol be used to examine first degree relatives and that caution be used in counselling apparently unaffected members of families at risk for TS.

Tuberous sclerosis results from a dominant gene which exhibits markedly variable expression and reduced penetrance. Reports attempting complete ascertainment indicate the prevalence to be as high as one in 15000 at birth. ${ }^{1}$

TS manifests clinically by symptoms or signs resulting from hamartomata in one or more organ systems, notably the skin and its appendages, the central nervous system, retina, heart, kidney, bones, and lungs. ${ }^{2}$ The classical skin manifestation is the facial rash of adenoma sebaceum. Other skin

Received for publication 5 February 1989.

Revised version accepted for publication 8 May 1989. findings may include hypopigmented macules (ash leaf spots), shagreen patches, and forehead fibrous plaques. ${ }^{3}$ The most serious features include mental retardation, fits, and intracerebral tumours. Findings in others systems include retinal hamartomata, cardiac rhabdomyomata, ${ }^{45}$ renal angiomyolipomata or cysts, and enamel pits. ${ }^{6-8}$

Although diagnostic criteria for TS have been suggested by Gomez, ${ }^{29}$ the variability of expression of the TS gene, reports consistent with reduced penetrance of the gene,$^{1011}$ or possible gonadal mosaicism of one of the parents ${ }^{12-16}$ can cause difficulty in genetic counselling, as persons without 
TABLE 1 Protocol for investigating first degree relatives of persons with TS.

(1) Complete physical examination with thorough examination of nail beds and skin including Wood's lamp

(2) Specialist ophthalmological examination by direct and indirect fundoscopy

(3) Specialist dental examination

(4) Cranial CT scan with and without contrast

(5) Echocardiography

(6) Renal ultrasound

(7) Skeletal survey

the obvious signs of TS could still be at risk of having affected children.

Early family studies done without full evaluation of the parents showed that only 10 out of 71 index cases had an affected parent. ${ }^{17}$ Subsequent family studies using skin, eye, and cranial CT scan showed nearly a half to be familial. ${ }^{18}$ More recently, Cassidy et al, ${ }^{19}$ using a more comprehensive, non-invasive screening protocol to look at apparently unaffected parents, showed the final proportion of familial cases to be just greater than $30 \%$.

In order to maximise the chance of detecting familial cases in apparently sporadically affected cases, we used a fully comprehensive, non-invasive protocol to screen parents and sibs of patients with TS so as to be able to give better advice to these relatives and to try to determine, if possible, which parts of the screening protocol are most effective.

\section{Methods}

Families were ascertained through paediatricians, neurologists, dermatologists, and ophthalmologists in the Yorkshire region, through the Tuberous

TABLE 2 Diagnostic criteria for TS as per Gomez ${ }^{2}$ $\left({ }^{*}\right.$ indicates changes since Gome $\left.{ }^{9}\right)$.

Pathognomonic (definitively diagnostic)

Adenoma sebaceum

Ungual fibroma

Retinal hamartoma, multiple or single*

Cortical tuber on pathology, $\mathrm{CT}^{*}$ or $\mathrm{MR}^{*}$

Multiple subependymal hamartoma on pathology, $\mathrm{CT}^{*}, \mathrm{MR}^{*}$

Multiple renal angiomyolipoma*

Forehead/scalp fibrous plaque*

Presumptive (provisionally diagnostic)

Infantile spasms

Depigmented patches

Shagreen patch

Single retinal hamartoma, peripapillary*

Multiple renal cysts

Cardiac rhabdomyoma on pathology or radiology*

Immediate relative with pathognomonic diagnosis of TS

Myoclonic, tonic, or atonic seizures*

Gingival fibroma*

Dental enamel pits

'Honeycomb' lung*

Multiple subcortical hypomyelinated lesions on $\mathrm{CT}^{*}$

Wedge shaped cortical/subcortical calcification on $\mathrm{CT}^{*}$
Sclerosis Association of Great Britain, and the genetic registry in our department and other genetic departments in the north of England.

Sixty-three families were contacted and of these 47 families had apparently sporadically affected subjects and 16 had affected subjects in two or three generations. Of these, 33 sporadically affected families and seven multigeneration families were willing to participate in the study.

Fifty-six first degree relatives from 32 families were evaluated by a fully comprehensive, noninvasive protocol (table 1). These consisted of 40 parents and seven sibs from 25 sporadically affected families and nine subjects from seven multigeneration families, five with affected sibs and parents, two with an affected sib but parental status was unknown or undetermined, and two parents from two families who on pedigree analysis were obligate heterozygotes. Three families were included for investigation even though the proband did not strictly fulfil Gomez's primary diagnostic criteria for TS. All three probands presented with fits and developmental delay. In addition, one proband had two depigmented patches and intracranial calcification on CT scan which was not thought to be typical of TS, the second had one depigmented patch and a white streak of hair, while the third had two depigmented patches.

All probands were reassessed by one of us (LIAG or RFM) using the pathognomonic or presumptive diagnostic criteria for TS as per Gomez. ${ }^{2}$ The same diagnostic criteria were also applied to the first degree relatives (table 2). For ethical reasons apparently unaffected at risk subjects under 16 years of age were not included in the study.

\section{Results}

FAMILIES (TABLE 3, FIG 1)

Of the 56 first degree relatives fully evaluated as described, 46 had a completely negative protocol while 10 had positive findings (table 4 ). The proband in eight of the families fulfilled Gomez's diagnostic

TABLE 3 Numbers of sporadic and multigeneration families with numbers of parents and sibs investigated and numbers with positive results on the screening protocol.

\begin{tabular}{lll}
\hline & Sporadic families & $\begin{array}{l}\text { Multigeneration } \\
\text { families }\end{array}$ \\
\hline Total No & 25 & 7 \\
Parents & 40 & 2 \\
Sibs & 7 & 7 \\
Positive parents & $8 / 40$ & $1 / 2$ \\
Positive sibs & $0 / 7$ & $1 / 7$ \\
Total positive & & \\
\hline
\end{tabular}


criteria for TS. Three mothers from three apparently sporadically affected families (pedigrees 1,2 , and 3 ) and one sib from one multigeneration family had echocardiographical evidence of rhabdomyoma (pedigree 4). One mother was found to have a typical area of calcification in the periventricular area on cranial CT scan (pedigree 5) and another was found to have bilateral renal cysts (pedigree 6). One father of a proband (pedigree 7) was found to have a tiny solitary density superior to the roof of the left lateral ventricle on cranial CT scan. In addition, he had bilateral bone islands in the necks of both femora.

In the two families where the proband did not even fulfil Gomez's provisional or presumptive diagnostic criteria for TS, the mothers of both these probands were found to have evidence of a rhabdomyoma on echocardiography (pedigrees 8 and 9).

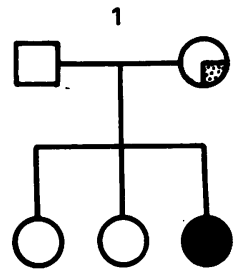

4

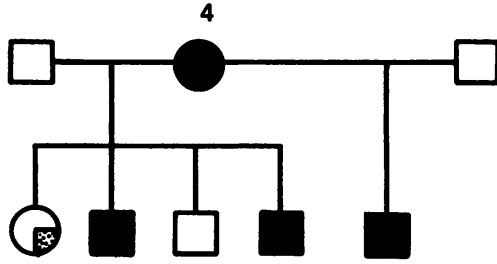

7

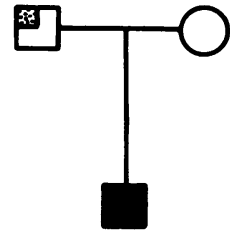

10

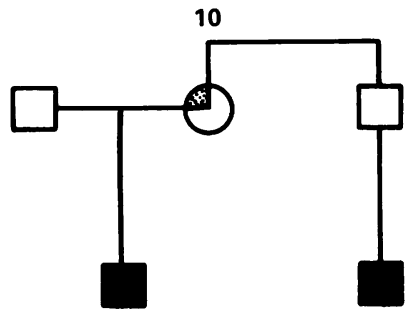

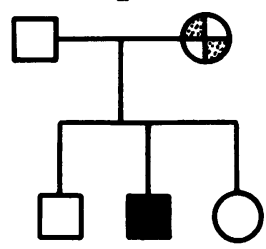

5

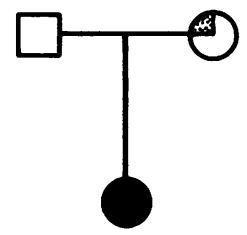

8

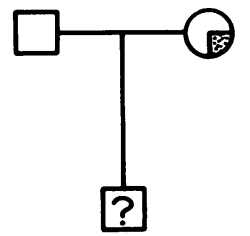

11

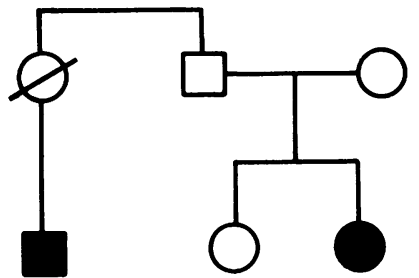

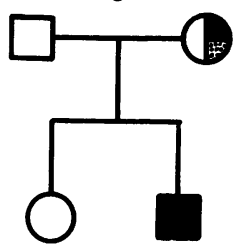

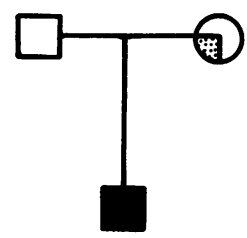

9

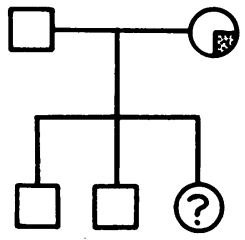

12

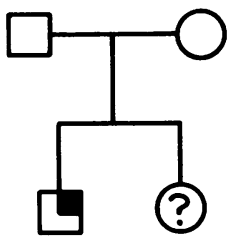


TABLE 4 Results of the various portions of the screening protocol positively identifying parents/sibs.

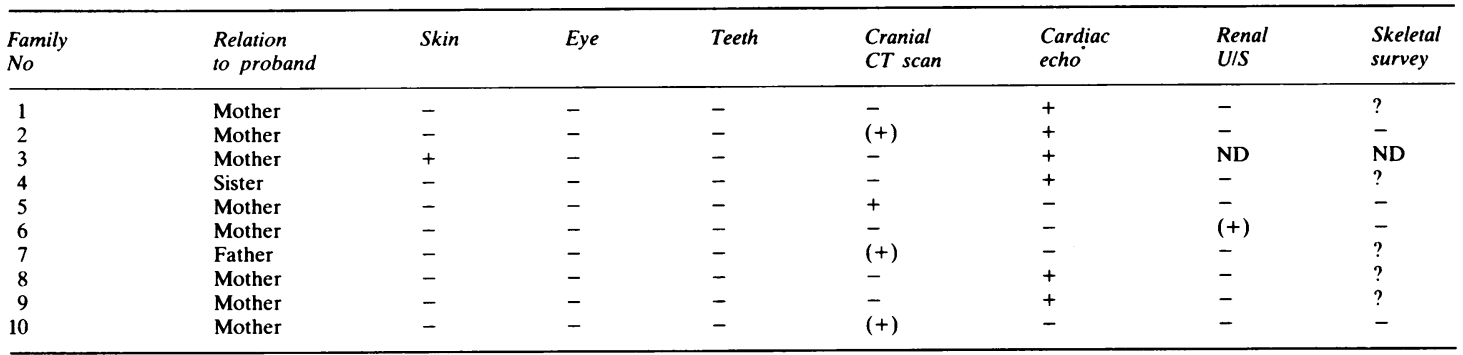

TABLE 5 Proportion of the various parts of the screening protocol positive.

\begin{tabular}{ll}
\hline Examination & Positive \\
\hline Skin & $1 / 56$ \\
Eye & $0 / 56$ \\
Teeth & $0 / 44$ \\
CT scan & $1(+3) / 56$ \\
Echo & $6 / 56$ \\
Renal U/S & $(1) / 55$ \\
Skeletal survey & $? / 52$ \\
\hline
\end{tabular}

One mother, an obligate heterozygote for TS, who had an affected child and nephew (pedigree 10) had as her only evidence of having the TS gene an area of enhancement in the medial wall of the left lateral ventricle on cranial CT scan. The second obligate heterozygote (pedigree 11) was completely normal on investigation by the protocol.

The brother of the proband who fulfilled provisional or presumptive diagnostic criteria for TS (pedigree 12) had typical depigmented patches but was not fully investigated because of his age.

\section{PROTOCOL (TABLE 5)}

\section{Cranial CT scans}

Cranial CT scans (unenhanced and contrast) were performed on all 56 first degree relatives, four being abnormal; one case was the mother who was an obligate heterozygote as mentioned. The CT scan of one mother showed a tiny area of increased density high up in the right frontal lobe (pedigree 2). This mother had, in addition, echocardiographical evidence of two rhabdomyomata (see below). A second mother showed a small focus of calcification in the left periventricular region (pedigree 5, fig 2). One father (pedigree 7) showed a tiny solitary area of increased density just above the roof of the left ventricle. In addition, he had bilateral bone islands in both femoral necks (see below).

\section{Echocardiography}

Of the 56 echocardiographs performed, six showed evidence of one or more rhabdomyoma. The proband in four families had definite diagnostic features of

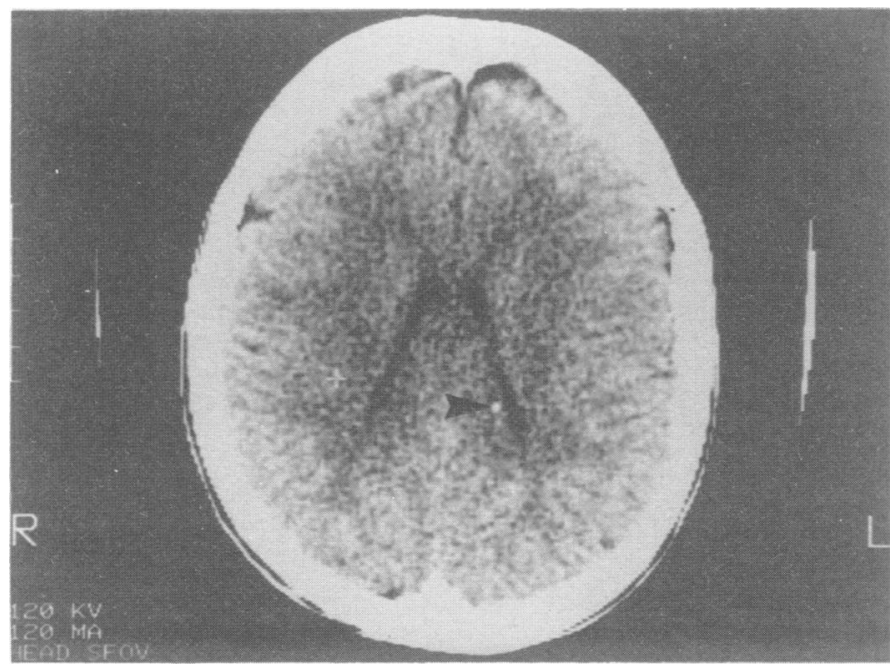

FIG 2 Unenhanced cranial CT scan of the mother in pedigree 5. There is a tiny calcified lesion in the left periventricular region (arrow). 


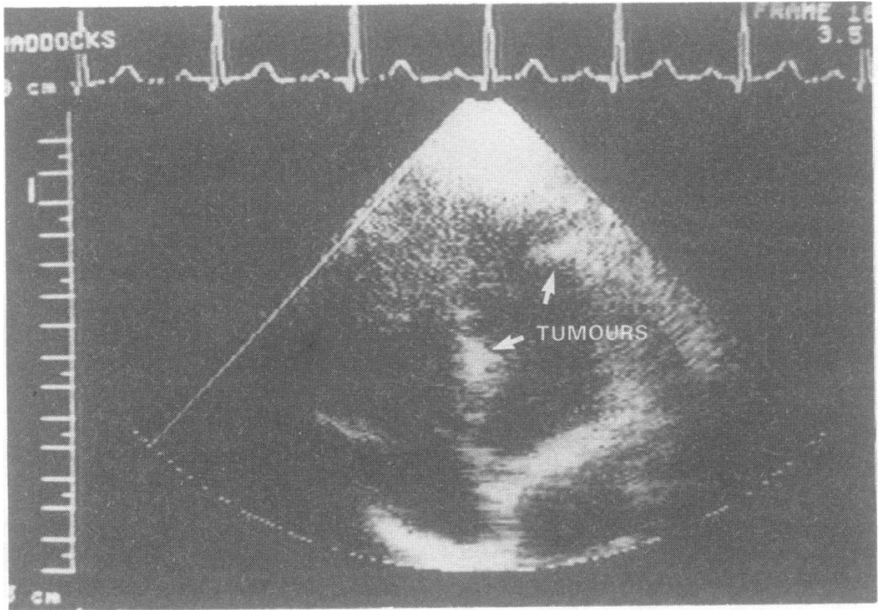

FIG 3 An apical four chamber echocardiographical view of the heart of the mother in pedigree 2 showing two rhabdomyomata, one in the central part of the interventricular septum and the other in the posterior left ventricular wall (as indicated).

TS. One mother of a proband (pedigree 2) had evidence of two rhabdomyomata, one $(10 \times 10 \mathrm{~mm})$ in the central part of the interventricular septum and one in the posterior left ventricular wall (fig 3). She was also found to have a positive cranial CT (as above). The mother of another proband (pedigree 3) was found to have two small 'echo dense' masses in the mid part of the interventricular septum, each $3 \mathrm{~mm}$ in diameter, and a further 4 to $5 \mathrm{~mm}$ diameter mass in the posterolateral apical left ventricular wall (fig 4). This mother also had two typical depigmented patches (see below). A third mother of a proband (pedigree 1) had a small subendocardial nodule $(5 \times 5 \mathrm{~mm})$ at the apex of the left ventricle (fig 5). A sib of a proband from a two generation family (pedigree 4) had evidence of a single, small $(5 \times 7.5 \mathrm{~mm})$ mass in the anterior wall of the right ventricle (fig 6). In addition, she had patchy sclerosis of the upper diaphyseal region of the right tibia and fibula and endosteal cortical thickening at the lower third of both femoral diaphyses (see below).

In the two instances where the proband did not fulfil Gomez's criteria for TS, the mother of one proband (pedigree 9) had evidence of a single subendocardial rhabdomyoma $(7 \times 8 \mathrm{~mm})$ adjacent to the medial mitral papillary muscle (fig 7). In addition, she had a lytic area ( $4 \mathrm{~mm}$ diameter) in the

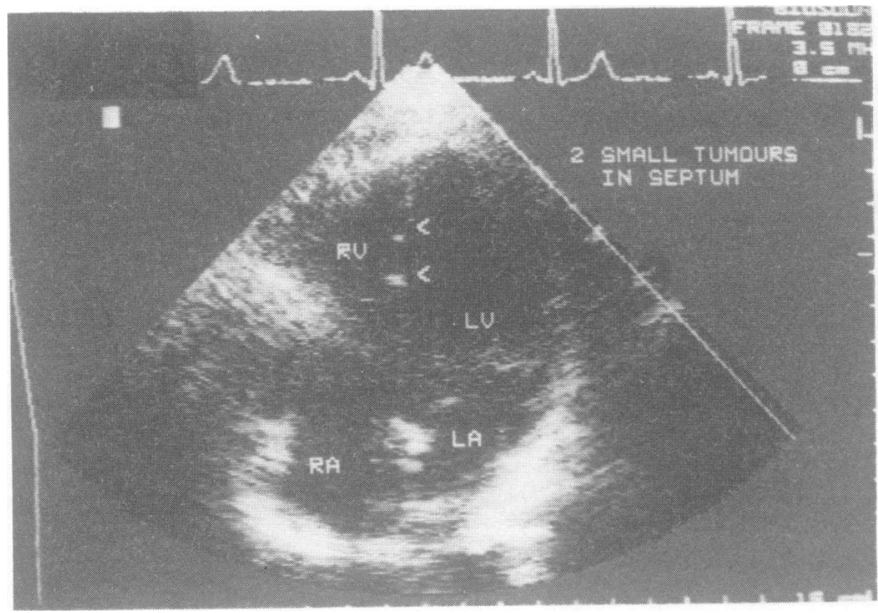

FIG 4 An apical four chamber echocardiographical view of the heart of the mother in pedigree 3 showing two small rhabdomyomata in the mid portion of the interventricular septum (as indicated). 


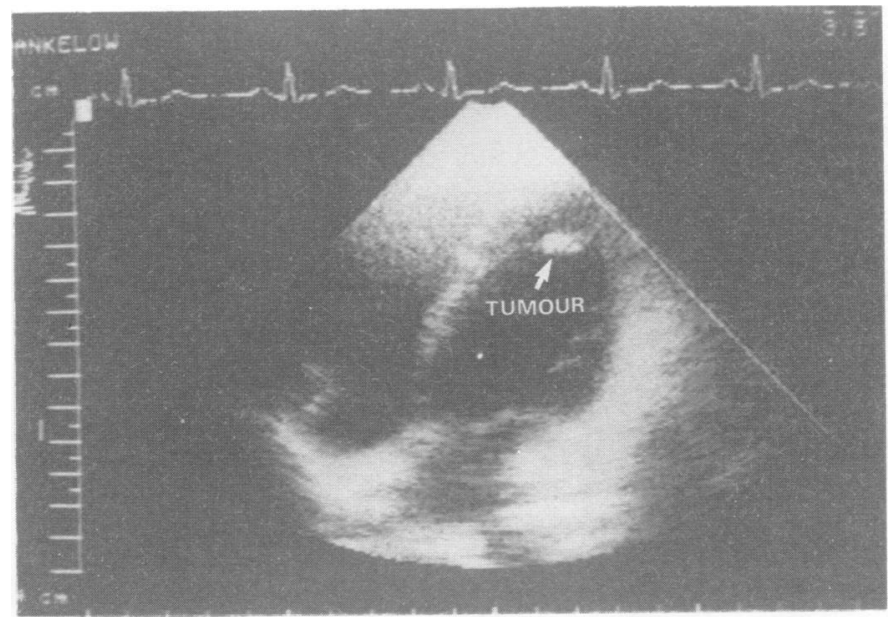

FIG 5 An apical four chamber

echocardiographical view of the heart of the mother in pedigree 1 showing a small subendocardial rhabdomyoma at the apex of the left ventricle (as indicated).

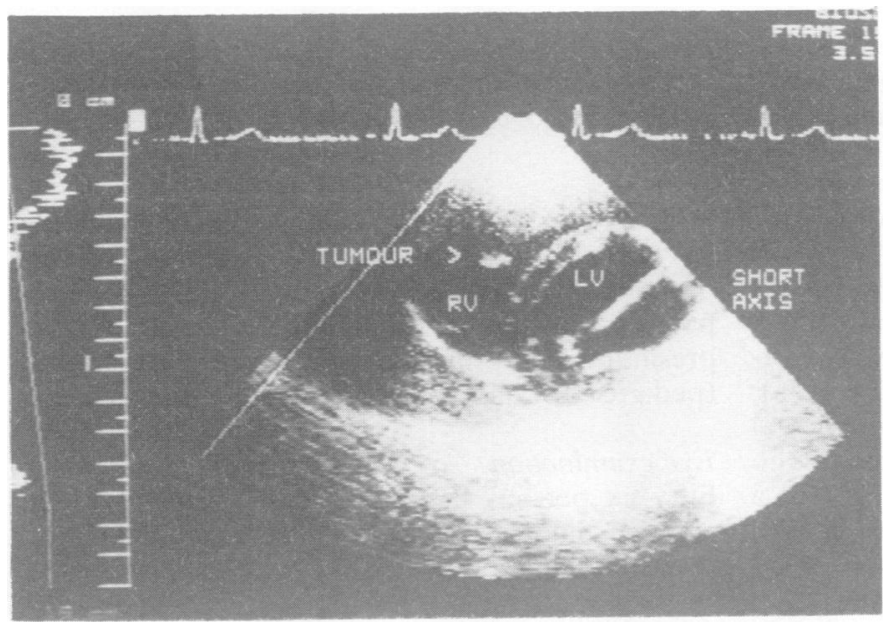

FIG 6 A short axis echocardiographical view of the heart of the sister in pedigree 4 showing a single small rhabdomyoma in the anterior wall of the right ventricle (as indicated).

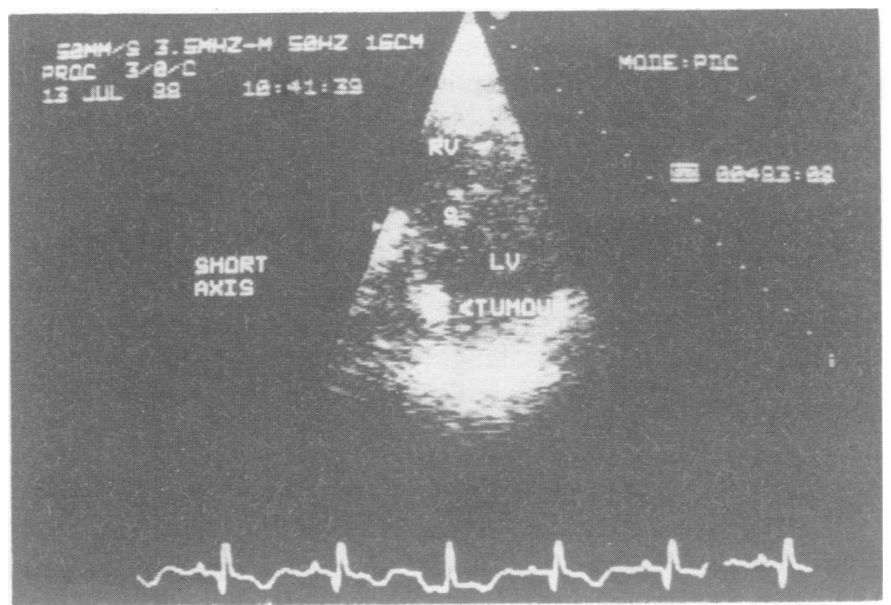

FIG 7 A short axis echocardiographical view of the heart of the mother in pedigree 9 showing a single subendocardial rhabdomyoma adjacent to the medial papillary muscle (as indicated). 


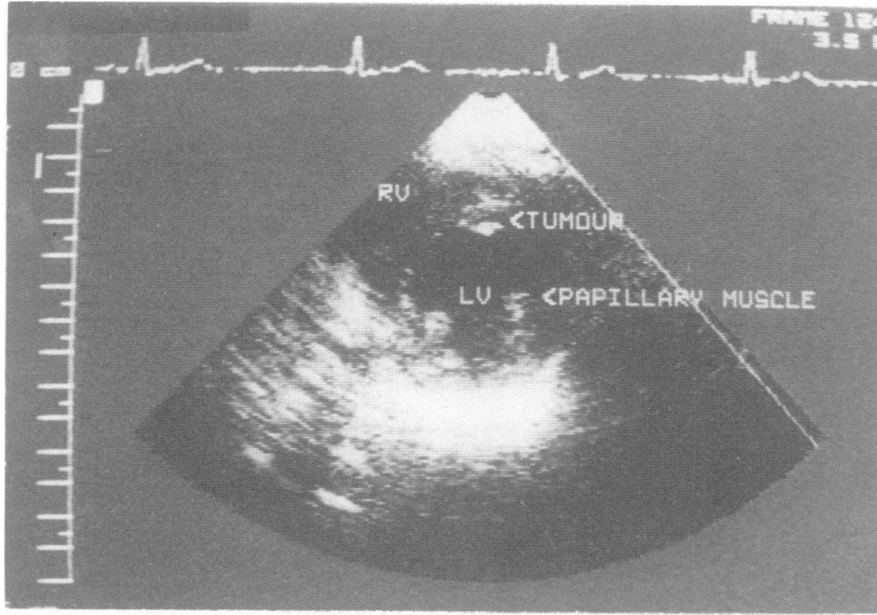

FIG 8 An apical four chamber echocardiographical view of the heart of the mother in pedigree 8 showing a small rhabdomyoma on the left ventricular aspect of the anterior part of the interventricular septum (as indicated).

middle phalanx of the right little finger (see below). The mother of the second proband (pedigree 8) had evidence of a small $(5 \times 5 \mathrm{~mm})$ rhabdomyoma on the left ventricular aspect of the anterior part of the interventricular septum (fig 8). In addition, she had a small cyst in the head of the proximal phalanx of the right little finger (see below).

\section{Renal ultrasound}

Fifty-five renal ultrasound examinations were performed. One mother, aged 34 years (pedigree 6), was found to have bilateral, single (1 $\mathrm{cm}$ diameter) renal cysts. Another mother, aged 36 years, who was an obligate heterozygote (pedigree 10), had a very small $(6 \mathrm{~mm}$ diameter) unilateral renal cyst.

\section{Skeletal survey}

Fifty-two skeletal surveys were performed; two parents had hand $x$ rays only, one mother was pregnant at the time, and the other refused this test. Positive findings included thickened skull vault (more than $1 \mathrm{~cm}$ ) in five persons and bone islands in 15 parents and sibs. The latter consisted of one bone island in five persons, two bone islands in five persons, three bone islands in two persons, and three persons with four, eight, and nine bone islands respectively. Nine parents and sibs had other nonspecific findings such as cysts and lytic areas which were thought to be degenerative cysts rather than the cystic changes reported as being seen in persons with TS. One parent had an abnormal trabecular pattern in his right femora and the sib with a positive echocardiogram (pedigree 4 ) had patchy sclerosis in her right tibia and fibula.

Although many of these skeletal findings are thought to be commonly present in persons with TS, in no single instance did the presence of any of these findings allow us to make the diagnosis of TS in an at risk relative.

\section{Skin}

Fifty-six persons had a thorough skin examination, all being normal apart from the mother of one proband who was found to have typical depigmented patches. The sib of the proband who only fulfilled presumptive or provisional diagnostic criteria for TS (pedigree 12) had typical depigmented patches.

\section{Eye examination}

Fifty-six persons had full ocular examination, including binocular indirect ophthalmoscopy with scleral indentation, all of which were normal.

\section{Dental examination}

Forty-four persons had specialist dental examination and five persons showed minimal enamel hypoplasia; however, none showed the specific enamel abnormalities described in persons with TS.

\section{Discussion}

The gene for TS exhibits variable expressivity and reduced penetrance ${ }^{2}$ which can lead to difficulties in genetic counselling. The importance of examining parents of affected subjects has been emphasised in a number of studies. Bundey and Evans ${ }^{17}$ found evidence of TS in earlier generations in 10 out of 71 families using clinical examination only. They concluded that the majority of cases of TS were examples of new mutations. Fleury et al, ${ }^{18}$ using skin, eye, and cranial CT scan to examine parents of 
TS patients, concluded that just under a half of cases were familial. Cassidy et al $^{19}$ recommended that parents of sporadically affected subjects should be evaluated by a protocol that included examination for the typical skin involvement, cranial CT scan, renal ultrasound, and dilated eye examination before recurrence risk counselling was given.

Three other non-invasive tests (echocardiography, skeletal survey, and dental examination) were added to the screening protocol of first degree relatives in this study as a significant proportion of persons with TS are reported to have findings in these systems. $^{2458}$ A more comprehensive protocol might also be expected to detect a greater proportion of familial cases allowing better advice to family members at risk. In addition, as this protocol is fairly time consuming and relatively expensive, it was hoped that the present study might determine which investigations are most effective for screening first degree relatives in order to give genetic advice.

Several studies have emphasised the value of cranial CT scan in detecting evidence of the TS gene in apparently unaffected relatives. Murphy et $a l^{20}$ reported a CT scan showing subependymal calcification in an asymptomatic mother of a child with TS. Cassidy et $a l^{19}$ found evidence of TS on cranial CT scan and renal ultrasound in one parent without manifestations in any other systems. Flinter and Neville ${ }^{15}$ evaluated eight families with skin examination and cranial CT scan and found that in two families one parent had a typical area of calcification in the absence of skin involvement. However, Racy et $a l^{21}$ evaluated nine first degree relatives of four children with TS by cranial CT scan and clinical examination and found no evidence of TS in any of them.

In the present study, cranial CT scan was definitely positive in one parent who was otherwise normal on examination by all other means (pedigree 5). In three other families, the CT scan findings were equivocal. One was the parent who is an obligate heterozygote (pedigree 10). In the other two families, the mother of the proband in one also had a positive echocardiogram (pedigree 2 ) and the father of the proband in the other had bone islands in both femora (pedigree 7). After this study, the latter family has had a second child who presented with infantile spasms at the age of four months.

Although the number of positive cranial CT scans as a proportion of the total number of first degree relatives evaluated is small, it can on occasion provide definitive information in isolation and may corroborate suggestive findings in other systems, which in themselves are not diagnostic of TS. The experience in this study suggests that these could be limited to non-contrast examination. There is evi- dence that magnetic resonance imaging has a useful role in the detection of the intracranial lesions of TS. ${ }^{22}{ }^{23}$ MR imaging is valuable in the detection of cortical tubers. However, CT is probably superior for screening family members at risk for TS owing to its greater sensitivity in the detection of small areas of intracranial calcification. ${ }^{24}$

Although rhabdomyomata have previously been thought to be a rare manifestation of the TS gene, recent studies have shown them to be present in a significant proportion of persons diagnosed by involvement of other systems as having TS. ${ }^{45}$ In a recent study, Smith $e t a l^{25}$ evaluated 37 first degree relatives ( 33 adults and four children) with echocardiography and skin examination. No cardiac tumours were identified in the adults but one child had two tumours in the left ventricle, with no other signs of TS. Two other children had rhabdomyomata and no other sign of TS when first seen, but developed signs in other systems at a later stage. The authors suggested that rhabdomyomata in TS may decrease in size with age and eventually disappear accounting for their apparent rarity in adults.

In our study, five parents and one sib (pedigrees 1 to 4,8 , and 9) had evidence of one or more rhabdomyomata on echocardiography. The apparently high prevalence seen in the first degree relatives in this study could be accounted for if these occurred commonly in the general population. Experience of echocardiography in a busy routine cardiac clinic suggests that these are a very rare finding. The observation of rhabdomyomata in two first degree relatives who have evidence in other systems consistent with or suggestive of having the TS gene means that echocardiographical findings of a rhabdomyoma occurring in isolation in a relative of a person with known TS should be taken seriously. Further studies screening first degree relatives of persons with TS by echocardiography will need to be done to evaluate the validity of this assertion. In the interim we feel this investigation should be included in any comprehensive evaluation protocol of relatives at risk.

Only one parent was found to have renal evidence of having the TS gene (pedigree 6). This finding is not sufficient to make a diagnosis of TS because single renal cysts are a common finding in the general population. ${ }^{26}$ Only biopsy of the wall of the cyst would allow a definite diagnosis.

The osseous findings in persons with TS have been reported to include multiple areas of sclerosis, especially in the calvarium, spine, and pelvis, cystlike lesions, and periosteal new bone formation in the hands and feet. ${ }^{27}$ Review of published reports of skeletal findings in persons with TS does not allow 
one to determine how frequently such findings occur in the general population. ${ }^{2}$ Although in a number of first degree relatives in this study one or more of these findings were observed, we were unable to use these to make a diagnosis of TS. Of interest, however, is the presentation of a second child with TS to the parents of an apparently sporadic case of TS (pedigree 7); the father had bone islands in both femoral necks along with cranial CT scan findings which were suggestive but not diagnostic of TS. Skeletal findings can be of little clinical usefulness in screening persons at risk for TS until a proper age/sex matched control study is carried out.

The eye examination in all of the persons examined was normal. Fleury et $a l^{18}$ noted positive eye findings in six first degree relatives, four of whom had no other evidence of TS. However, it has been suggested that it is extremely uncommon for retinal phakomata to be the only sign of TS. ${ }^{28}$

Cassidy et $\mathrm{al}^{19}$ found positive skin findings in three parents of persons with TS, concluding that careful and detailed skin examination was the most sensitive diagnostic test. In this study only one parent was found to have skin findings diagnostic of TS.

Lygidakis and Lindenbaum ${ }^{8}$ found multiple typical dental pits in one parent and one half sib of persons with TS who were without any other signs of the disorder, and recommended that dental examination for pitted enamel hypoplasia might identify persons otherwise unsuspected of having the TS gene in families at risk. However, in the present study none of the parents or sibs examined was found to have any evidence of other than minimal enamel pitting in five cases.

There have been many reports of apparent nonpenetrance in TS. ${ }^{10-16}$ In all these reports, apart from that of Connor et al, ${ }^{14}$ the families were investigated to a limited extent. The report of apparent non-penetrance by Connor et $\mathrm{l}^{14}$ included all the investigations in our protocol apart from echocardiography. In our study, two families exhibited apparent non-penetrance (pedigrees 10 and 11). Of interest is the fact that the parents in pedigree 11 are first cousins. The brother of the proband in pedigree 12 had typical depigmented patches but was not investigated fully because he was under age.

Gomez established diagnostic criteria for TS in $1979^{9}$ allowing affected subjects to be diagnosed with confidence. In his revised diagnostic criteria, Gomez $^{2}$ put radiological evidence of rhabdomyoma as presumptive or provisionally diagnostic for TS. The usefulness of this and other presumptive diagnostic criteria for TS is still not clear as the prevalence of these features in the general population is not known.
Although in 10 families investigated, a parent or sib had one or two positive findings, we could give definitive genetic advice in only two families (pedigrees 3 and 5). In one family (pedigree 5) the mother had a positive cranial CT scan while in the second family the mother had depigmented patches and echocardiographical evidence of rhabdomyomata (pedigree 3). It was very difficult to give definite genetic advice in the remaining seven families. In four families the proband fulfilled Gomez's criteria, but the findings in the parents and sibs did not satisfy diagnostic criteria for TS. One mother (pedigree 1) and a sister (pedigree 4) had a positive echocardiogram, the latter also having sclerosis of the upper diaphyseal region of the tibia. Another mother (pedigree 2) had a positive echocardiogram and a tiny area of increased density on the cranial CT scan. Another mother (pedigree 6) was found to have bilateral single renal cysts.

In two families (pedigrees 8 and 9) the proband did not strictly fulfil Gomez's diagnostic criteria for TS although both mothers had echocardiographical evidence of rhabdomyoma. In a third family, the proband (pedigree 12) had depigmented macules, fits, and mental retardation, fulfilling provisional diagnostic criteria for TS. Her brother had multiple depigmented macules, but because his sister did not fulfil the pathognomonic diagnostic criteria, a definitive diagnosis of TS could not be made in him.

The extent to which first degree relatives of persons with TS are investigated when providing genetic counselling varies in different centres. In part this can be attributed to the fact that it is not clear which of the many investigations which could be carried out are the most useful in determining who has the gene for this disorder.

The various reports of non-penetrance of the TS gene make it difficult to reassure completely relatives of apparently sporadically affected subjects that further persons will not be at risk. From our study there does not appear to be a single test or set of tests which can be used reliably in isolation and we suggest that first degree relatives of TS patients should be evaluated by an extensive non-invasive protocol. While there is likely to be a minimal yield of positive findings from such a protocol, the absence of abnormal findings could be used as positive evidence of the likelihood of a person at risk not having the TS gene. If more detailed information were available on the prevalence of those features commonly present in persons with TS in the general population, this could be used in a Bayesian calculation to give more accurate risk figures. In the interim we urge caution in counselling families at risk for TS. 
We thank the Tuberous Sclerosis Association of Great Britain for funding this project and all the families who participated in this study.

\section{References}

1 Hunt A. Lindenbaum R. Tuberous sclerosis: a new estimate of prevalence within the Oxford region. J Med Genet 1984;21: $272-7$.

2 Gomez MR. Tuberous sclerosis. 2nd ed. New York: Raven Press, 1988.

${ }^{3}$ Fryer AE, Osborne JP, Schutt W. Forehead plaque: a presenting sign in tuberous sclerosis. Arch Dis Child 1987;62:292-304.

${ }^{4}$ Bass JL, Breningstall GN, Swaiman KF. Echocardiographic incidence of cardiac rhabdomyoma in tuberous sclerosis. Am J Cardiol 1985:55:1379-82.

${ }^{5}$ Gibbs JL. The heart and tuberous sclerosis. An echocardiographic and electrocardiographic study. Br Heart $J$ 1985;54: 596-9.

${ }^{6}$ Hoff M. van Grunsven MF. Jongbloed WL. Gravenmade EJ. Enamel defects associated with tuberous sclerosis. Oral Surg Oral Med Oral Pathol 1975;40:261-9.

7 Weits-Binnerts JJ, Hoff M, Van Grunsven MF. Dental pits in deciduous teeth, an early sign in tuberous sclerosis. Lancet $1982 ; \mathrm{ii}: 1344-5$.

${ }^{8}$ Lygidakis NA. Lindenbaum RH. Pitted enamel hypoplasia in tuberous sclerosis patients and first-degree relatives. Clin Genet 1987;32:216-21.

${ }^{9}$ Gomez MR. Tuberous sclerosis. New York: Raven Press, 1979.

${ }^{10}$ Rushton AR, Shaywitz BA. Tuberous sclerosis: possible modification of phenotypic expression by an unlinked dominant gene. J Med Genet 1979;16:32-5.

1 Baraitser M, Patton MA. Reduced penetrance in tuberous sclerosis. J Med Genet 1985;22:29-31.

12 Wilson J, Carter C. Genetics of tuberous sclerosis. Lancet 1978;i:340.

${ }^{13}$ Lowry RB, Dunn HG, Paris RP. Inheritance of tuberous sclerosis. Lancet 1979;i:216.

${ }^{14}$ Connor JM, Stephenson JBP, Hadley MDM. Non-penetrance in tuberous sclerosis. Lancet 1986;ii: 1275.
15 Flinter FA, Neville BGR. Examining the parents of children with tuberous sclerosis. Lancet 1986;ii: 1167.

${ }^{16}$ Berberich MS. Hall B. Penetrance and variability in tuberous sclerosis. Birth Defects 1979:XV(5B):297-304.

${ }^{17}$ Bundey S. Evans K. Tuberous sclerosis. J Neurol Neurosurg Psychiatry 1969:32:591-603.

18 Fleury P, de Groot WP, Delleman JW, Verbeeten B, Frankenmolen-Witkiezwicz I. Tuberous sclerosis: the incidence of sporadic versus familial cases. Brain Dev 1980;2:107-17.

19 Cassidy SB. Pagon RA. Pepin M. Blumhagen JD. Family studies in tuberous sclerosis. Evaluation of apparently affected parents. JAMA 1983:249:1302-4.

20) Murphy JV. D Souza BJ, Haughton VM. CT scan and tuberous sclerosis. JAMA 1976;236:115.

${ }^{24}$ Racy A, Barakat AY, Cochran WE. Computerized axial tomography in family members of patients with tuberous sclerosis. Clin Pediatr (Phila) 1978;17:883-5.

${ }^{22}$ McMurdo SK, Moore SG, Brant-Zawadzki M. Bergo BO, Newton TH, Edwards MSB. MR imaging of intracranial tuberous sclerosis. AJNR 1987;8:77-82.

${ }^{23}$ Roach ES, Williams DP, Laster DIV. Magnetic resonance imaging in tuberous sclerosis. Arch Neurol 1982;44:301-3.

24 Altman NR, Purser RK, Donovan JD. Tuberous sclerosis: characteristics at CT and MR imaging. Radiology 1988;67: 527-32.

${ }^{25}$ Smith H, Patel RG. Watson GH. Cardiac rhabdomyomata in tuberous sclerosis. Arch Dis Child 1989:64:196-200.

${ }^{26}$ Tada S. Yamagishi J. Kobayashi H. Hata Y. Kobari T. The incidence of simple renal cysts by computed tomography. Clin Radiol 1983;34:437-9.

27 Holt J. Dickerson W. The osseous lesions of tuberous sclerosis. Radiology 1952;58:1-7.

${ }^{2 x}$ Osborne JP. Diagnosis of tuberous sclerosis. Arch Dis Child 1988;63:1423-5.

Correspondence to Dr L I Al-Gazali, Department of Genetic Counselling, Clarendon Wing, The General Infirmary, Belmont Grove, Leeds LS2 9NS. 